\title{
Optical Plume Velocimetry: A New Flow Measurement Technique for Use in Seafloor Hydrothermal Systems
}

Received: date / Accepted: date

\begin{abstract}
Evidence suggests that fluid flow rates in mid-ocean ridge hydrothermal systems may be strongly influenced by mechanical forces such as ocean tidal loading. However, long time-series measurements of flow have not been collected in these environments. We develop a non-invasive method, called optical plume velocimetry (OPV), suitable for obtaining fluid flow rates through black smoker vents based on image analysis of effluent video. We use video from laboratory flows to evaluate three different methods for estimating the image-velocity field that are based on region-based matching, spectral-analysis of Hovmöller diagrams, and temporal cross-correlation of adjacent pixel values. We find that OPV is most sensitive and least biased when the cross-correlation method is used and conclude that that OPV should not be applied to flows that are transitioning between jet-like and plume-like behavior.
\end{abstract}

Keywords Black smoker plumes $\cdot$ Hydrothermal vents $\cdot$ Optical velocimetry $\cdot$ Flow rate measurement

\section{T. J. Crone}

Columbia University

Lamont-Doherty Earth Observatory

61 Route 9W

Palisades, NY 10964-8000

E-mail: crone@1deo.columbia.edu

R. E. McDuff · W. S. D. Wilcock

University of Washington

School of Oceanography

Box 357940

Seattle, WA 98195-7940 


\section{List of Symbols}

A $\quad$ area of jet nozzle, $\mathrm{m}^{2}$

$B \quad$ initial specific buoyancy flux, $\mathrm{m}^{4} / \mathrm{s}^{3}$

$c_{1} \quad$ constant in along-axis plume velocity equation

$c_{f} \quad$ fraction of Nyquist frequency for Hovmöller cut-off

C temporal cross-correlation function

$D \quad$ jet nozzle diameter, $\mathrm{m}$

$d \quad$ pixel separation in temporal cross-correlation method, pixels

$f \quad$ frequency, $1 / \mathrm{s}$

$g$ gravitational acceleration, $\mathrm{m} / \mathrm{s}^{2}$

$k_{1} \quad$ constant in along-axis jet velocity equation

$l_{M} \quad$ Morton length scale, $\mathrm{m}$

$l_{\max }$ lag number at the cross-correlation maximum, frames

$M \quad$ initial specific momentum flux, $\mathrm{m}^{4} / \mathrm{s}^{2}$

$n \quad$ number of instantaneous image-velocity measurements used to calculate the mean

$N \quad$ number of frames in image sequence

$Q \quad$ nozzle flow rate, $\mathrm{m}^{3} / \mathrm{s}$

$Q_{i} \quad$ individual nozzle flow rate measurement, $1 / \mathrm{s}$

$Q_{m} \quad$ mean measured nozzle flow rate, $1 / \mathrm{s}$

$r \quad$ radial coordinate, $\mathrm{m}$

$R \quad$ Residual for region-based matching image-velocity estimation

Re Reynolds number

$S \quad$ standard deviation

$\Delta t_{10} \quad$ time for source fluid tank level to drop by 10 liters, $\mathrm{s}$

$\bar{u} \quad$ flow velocity, $\mathrm{m} / \mathrm{s}$

$t \quad$ time, $\mathrm{s}$

$u_{p} \quad$ instantaneous image-velocity, pixels/frame

$\overline{u_{p}} \quad$ mean image-velocity, pixels/frame

$U_{m} \quad$ mean along-axis jet velocity, $\mathrm{m} / \mathrm{s}$

$U_{p} \quad$ flow rate metric, pixels/frame

$W$ mean flow velocity across jet nozzle, $\mathrm{m} / \mathrm{s}$

$W_{p} \quad$ mean flow velocity across jet nozzle converted to pixels per second, pixels/frame

$x \quad$ horizontal coordinate, $\mathrm{m}$ 
$x_{p} \quad$ projected horizontal coordinate, pixels

$z \quad$ vertical coordinate, $m$

$z_{p} \quad$ projected vertical coordinate, pixels

\section{Greek Symbols}

$\lambda_{p} \quad$ flow feature wavelength, pixels

$v \quad$ Hovmöller FFT wave number, 1/pixels

$\mu \quad$ viscosity of jet fluid, Pa s

$\rho \quad$ density of ambient fluid, $\mathrm{kg} / \mathrm{m}^{3}$

$\rho_{J} \quad$ density of jet fluid, $\mathrm{kg} / \mathrm{m}^{3}$

$\Delta \rho \quad$ density difference between jet and ambient fluid, $\mathrm{kg} / \mathrm{m}^{3}$

\section{Introduction}

Aqueous fluid flow within mid-ocean ridge hydrothermal systems is responsible for large fluxes of heat and chemicals between young oceanic crust and the ocean (Elderfield and Schultz 1996). These fluxes have wide-ranging effects, as they influence the physical properties of the lithosphere, the chemical composition of the crust and ocean, and the biological systems at and below the seafloor (Kelley et al 2002). The seafloor expression of these hydrothermal systems are vent fields where hydrothermal fluids enter the ocean after undergoing thermal convection in the lithosphere (e.g., Alt 1995). When the hottest fluids mix with cold seawater, dissolved metals and minerals precipitate to form chimney-like geological structures that channel flow into the ocean. These precipitates give the fluids the appearance of black-colored smoke, hence these vents are often called "black smokers".

Black smokers can take on a variety of morphologies, from spires to mounds, and can vary significantly in height with some standing only a meter or so tall and others standing tens of meters tall (Delaney et al 1992; Spiess et al 1980). The flow through these structures is often vigorous and turbulent, with typical flow rates on the order of $\sim 0.5-2 \mathrm{~m} / \mathrm{s}$ (e.g., Converse et al 1984). The orifices through which fluids exit range in diameter from about $0.02-0.1 \mathrm{~m}$ (e.g., Converse et al 1984). Fluid temperatures are typically $350-400^{\circ} \mathrm{C}$, and at typical seafloor pressures these fluids can have densities below $650 \mathrm{~kg} / \mathrm{m}^{3}$ (Bischoff and Rosenbauer 1985).

Models and indirect measurements suggest that black smoker flow is highly variable and may be strongly influenced by tidal, tectonic, and magmatic processes (Butterfield et al 1997; Crone and Wilcock 2005; Crone et al 2006; Davis et al 2004; Delaney et al 1998; Johnson et al 2000; Larson et al 2007; 
McDuff and Delaney 1995; Tolstoy et al 2002; Wilcock and Fisher 2004). A small number of direct measurements in diffuse flows confirm this possibility (e.g., Jupp 2000; Pruis and Johnson 2004; Schultz et al 1996), as do direct visual observations (M. Lilley, pers. comm.). The potential sensitivity of subseafloor flow to mechanical forces suggests that measurements of black smoker flow may be critical to understanding the links between the physical, chemical, and biological components of these systems.

Despite the potential importance of flow measurements in these environments, no long time-series records of black smoker flow rates exist. High fluid temperatures, low $\mathrm{pH}$, and mineral deposition render time-series measurements using invasive measurement techniques viable only over timescales on the order of minutes (e.g., Converse et al 1984). Non-invasive techniques used in other settings, such as laser Doppler velocimetry (Coupland 2000) and acoustic Doppler velocimetry (Kraus et al 1994) have never been used on black smoker flows, and their implementation may be complicated by the extreme optical and acoustic property differences between black smoker fluids and ambient seawater.

Another potential non-invasive technique for measuring black smoker fluid flow utilizes video image analysis, whereby time-averaged image-velocity fields computed from imagery of black smoker effluent are used to estimate nozzle flow rates. One motivation for developing an image-based measurement technique is the existence of numerous historical video data sets showing black smoker flows over time periods of weeks and months (McDuff and Delaney 1995). Optical plume velocimetry (OPV) could be used to explore these data for flow variability associated with tidal and tectonic activity. An ultimate goal would be to incorporate OPV into future seafloor instrumentation that could be deployed as part of a seafloor cabled observatory (e.g., Delaney et al 2000).

In this paper we describe the development of an image-based technique for estimating flow rates through black smoker hydrothermal vents. The steps we follow are outlined in Figure 1, which is a conceptual flow diagram highlighting a number of issues that need to be considered. We begin by discussing the relevant theory of turbulent jets and plumes, and then we describe the laboratory experiments used to obtain video data of simulated black smoker jets with known flow rates. We describe the three computational techniques used to estimate relative nozzle flow rates from these video data, and we detail the results from each technique. We conclude with a discussion of the results and the implications for the development of seafloor instrumentation and the analysis of historical data.

\section{Theory of Turbulent Buoyant Jets and Plumes}

A key consideration for the development of an image-based flow measurement technique is the evolution of constrained nozzle flow into a fully-developed free turbulent flow (Transform A in Figure 1). Black 
smoker hydrothermal vents produce turbulent buoyant jets, which are flows that have an initial momentum flux and an initial buoyancy flux at the jet nozzle (McDuff 1995). To simplify our discussion, we will treat black smoker flows as round radially-symmetric turbulent buoyant jets. Because we are interested in flow variability that occurs on timescales that are much longer than the timescales of the turbulence, we need only consider the time-averaged mean properties of these flows. The theory we follow was originally proposed by Morton et al (1956) and is reviewed in detail by List (1982) and Turner (1986). Many experimental studies have added to our understanding of turbulent buoyant jets and constrained many of the relevant parameters (Papanicolaou and List 1988; Shabbir and George 1994; Ruden 1933).

Near the nozzle, turbulent buoyant jets behave like pure jets, which are flows created by a pressure drop across an orifice (List 1982), and have no buoyancy flux associated with them. Such flows, when they are steady, develop self-similarity at some distance from the orifice and have mean properties that can be entirely described by a single parameter. In the case of pure jets, this parameter is the specific momentum flux at the nozzle, $M$, where (McDuff 1995):

$$
M=Q W=A W^{2},
$$

and $Q$ is the nozzle flow rate, $W$ is the mean nozzle velocity, and $A$ is the area of the nozzle opening. The mean velocity of the flow along the jet axis, $U_{m}$, as a function of distance from the virtual origin, $z$, is given by (Turner 1986):

$$
U_{m}=k_{1} M^{1 / 2} z^{-1}
$$

where $k_{1}$ is a constant that has been found experimentally to be $\sim 7$, and the virtual origin is some distance inside the nozzle. The mean velocity as a function of the radial distance from the jet axis, $r$, is given by (Turner 1986):

$$
\bar{u}=U_{m} \exp \left[-\left(\frac{r}{b_{u}}\right)^{2}\right]
$$

where $b_{u}$ is a constant that has been found experimentally to be $\sim 0.1 z$. Because $Q$ is equal to $W$ multiplied by the area of the jet nozzle, these equations show that mean velocities everywhere in a fully-developed pure jet are proportional to $W$.

Far from the nozzle, turbulent buoyant jets behave like pure plumes. Pure turbulent plumes usually arise from density differences between the plume fluid and the ambient fluid, and have no initial momentum flux (Rodi 1982). Like pure jets, pure plumes become self-similar away from the nozzle, and have mean properties that can be entirely described by a single parameter. In this case the parameter is the specific 
buoyancy flux at the nozzle, $B$, where (Papanicolaou and List 1988):

$$
B=g \frac{\Delta \rho}{\rho} Q,
$$

and $g$ is the gravitational acceleration, $\Delta \rho$ is the density difference between the plume and ambient fluid, and $\rho$ is the density of the ambient fluid. The mean velocity along the plume axis is given by (Turner 1986):

$$
U_{m}=c_{1} B^{1 / 3} z^{-1 / 3}
$$

where $c_{1}$ is a constant that has been found experimentally to range from 3.4-3.9 (List 1982; Papanicolaou and List 1988). The mean velocity, $\bar{u}$, as a function of the radial distance from the jet axis, $r$, has a similar form as that for a pure jet (Papanicolaou and List 1988). Thus for fully-developed pure plumes, the velocity is predicted to be proportional to $W^{1 / 3}$.

Over some range of distances from the nozzle, turbulent buoyant jets transition between the pure jet and pure plume end-members. This transition occurs because in a pure jet, the integrated momentum flux across any plane perpendicular to the jet axis is fixed, whereas a plume is continually gaining momentum, as it started with none. At some distance from the nozzle the plume momentum will overwhelm the jet momentum, and the flow will begin to behave like a pure plume. The dimensionless distance $z / l_{M}$ characterizes this transition, where $l_{M}$ is the Morton length scale, given by (Turner 1986):

$$
l_{M}=\frac{M^{3 / 4}}{B^{1 / 2}}
$$

Flows behave like jets when $z / l_{M}$ is less than unity, and they behave like plumes when $z / l_{M}$ is greater than about 5. In between, the flow is transitional (Papanicolaou and List 1988).

Transitional flows are less well-understood than the two end-member flows, but what is certain is that mean velocities in this part of the flow transition from being proportional to $W$ to being proportional to $W^{1 / 3}$. For this reason the functional form of the relationship between these quantities is dependent upon position. Further, because the distances of transitional flow is a function of flow rate, the functional relationship between these quantities at any single position is also dependent on flow rate. These are key issues in regard to image-based flow measurement because any uncertainty in the functional relationship between $\bar{u}$ and $W$ will complicate attempts to ascertain nozzle flow rates using optical methods. Imagebased measurement techniques should not rely heavily on imagery of transitional flows.

One complex but important consideration for OPV is the relationship between the three-dimensional turbulent velocity field and the visible flow field, for they are not equivalent (Transform B in Figure 1). 
The visible portion of the flow at any one time is dependent upon lighting, tracer composition and concentration, partial translucency of the flow structures, and the instantaneous size and shape of the turbulent flow structures near the jet boundary layer. Thus, when looking at any one part of the flow, a wide range of velocities might be observed not only because the fluid flow is turbulent but also because different locations within the flow are being sampled. We hypothesize that averaged over time the visible flow field is a spatial average of the fluid velocities from the outer edge of the flow to some distance into the flow. In this paper we refer to this distance as the "viewable depth".

\section{Laboratory Simulations}

In order to develop the OPV technique, we constructed a laboratory apparatus to simulate black smoker flows with known flow rates. We used a video camera to capture image sequences that contain twodimensional projections of the three dimensional flow field for our analysis (Transform C in Figure 1).

\subsection{Apparatus}

Our experimental setup for the black smoker jet simulations is shown in Figure 2. For each simulation run, fluid from the constant-head tank passed through a flow straightener and downward into a 2.1-m-tall main tank through a nozzle with diameter $18 \mathrm{~mm}$. The tank's cross-sectional size was $0.9 \times 0.9 \mathrm{~m}$. The jet fluid consisted of tap water with $\sim 0.5 \mathrm{wt} \% \mathrm{NaCl}$ to provide buoyancy flux, and colloidal graphite particles to simulate black smoker particulates. The main tank was filled with plain tap water. We adjusted the nozzle flow rate with flow constrictors of varying diameter and by changing the constant-head tank overflow height. With this arrangement we were able to produce mean nozzle velocities over the range $\sim 0.065-0.65 \mathrm{~m} / \mathrm{s}$, corresponding to Reynolds numbers of $\sim 1200-12000$, where $R e \equiv \rho_{J} W D / \mu$. On the upper end, this range was limited by the head height achievable with the apparatus.

We illuminated the flow in the test section with two submerged halogen flood lights, and used two thin sheets of red and blue dye-tinted acrylic for the background, which effectively absorbs much of the incident light. This lighting arrangement was designed to replicate conditions under which seafloor vent video is obtained. In the same plane as the jet flow, we placed a ruler with alternating black and white squares, $0.01 \mathrm{~m}$ on a side, for scale.

We recorded the simulations using a QImaging Retiga EX digital video camera capturing 33 frames per second with an image resolution of $332 \times 248$ pixels in 8-bit grayscale. Uncompressed data from this camera was streamed directly to the storage medium in the lossless AVI file format. This recording system 
created video files that were 30-s in length, containing 990 frames each. When switching files, the system dropped several frames (the exact number could not be determined), which limited our ability to complete certain types of analyses requiring longer uninterrupted sequences. However, for the analyses carried out in this paper, we found 30-s video clips to be sufficient in length. The relatively low spatial resolution of this camera system allowed us to evaluate the expected performance of OPV when applied to the historical data of similar resolution (McDuff and Delaney 1995), and when used with an underwater system that may have a low data rate.

To measure the nozzle flow rate during our simulations, we monitored the rate at which the fluid level decreased in the lower source tank, noting the time required for the fluid level to pass 10-liter graduation marks on the tank. For each run, we made 3-5 estimates of the nozzle flow rate. The uncertainty of this measurement technique resulting from the combination of uncertainty in timing and uncertainty in the tank's graduation marks was about $6 \%$.

\subsection{Simulations}

We conducted five simulations to obtain the video data used in this study, which we number 1-5. Figure 3 shows a single typical image frame from one of the simulations, and Movies 1-5, available as Electronic Supplementary Material, contain 30-s samples of the video collected during Simulations 1-5 respectively. In this paper we rotate images of the jet flow and the computed image-velocity fields so the $z$-axis is positive upward. Each simulation yielded about 9-12 minutes of usable video data, corresponding to about 17,000-24,000 images for each run.

Table 1 lists the measured nozzle flow rates for the laboratory simulations, and Table 2 lists derived flow rate data, including the mean flow velocity across the jet nozzle in meters per second and in pixels per frame. To compute the latter value we used a conversion factor of 858 pixels per meter, which we estimated using the scale placed next to the flow. Also listed in Table 2 are the corresponding nozzle Reynolds numbers for the simulations, which range from 2900 to 6700. These Reynolds numbers are 12 orders of magnitude lower than those of real black smokers, primarily because the viscosity of black smoker fluids are about an order of magnitude lower than water at standard temperature and pressure (Holzbecher 1998). However, Dimotakis et al (1983) show that when Reynolds numbers are sufficiently high and there are no neighboring solid boundaries that influence the flow, the Reynolds number does not influence the size of the largest scales of turbulence in these flows. The largest turbulent length scales in these flows are limited by the width of the plume, which does not change as a function of nozzle flow rate. 
Figure 4 shows the approximate size of the transitional flow field relative to the camera's field of view for each of the five simulations. While the imaging of transitional flow is not recommended for future studies of black smoker flow rates, in this study one of our primary motivations is the evaluation of this technique's potential performance when applied to historical video data which is likely to include transitional flow. Thus we generate simulated flow imagery with transitional flow, but we include little transitional flow in our analyses.

\section{Computational Methods}

\subsection{The Time-Averaged Image-Velocity Field}

The image-velocity field (also sometimes referred to as the optical flow field) is a two-dimensional vector field describing the motion of objects imaged within a video sequence (Transform D in Figure 1). The generation of an image-velocity field from an image sequence is a classic problem in the field of image analysis, and has been studied for nearly three decades (Beauchemin and Barron 1995). Solutions to this problem have applications in computer vision, robotics, automation, and artificial intelligence, as well as in the study of fluid dynamics.

There are several classes of optical flow calculation techniques, and many variants within each class (Beauchemin and Barron 1995). Commonly used techniques include: differential methods which rely on spatiotemporal derivatives of image intensity (e.g., Horn and Schunck 1981), frequency-based methods which are based on the output of velocity-tuned filters (e.g., Adelson and Bergen 1985), and region-based matching techniques which use spatial cross-correlation applied to features within the image (e.g., Anandan 1989). The study of fluid dynamics using image sequence analysis has largely relied on variants of region-based matching, including particle image velocimetry (e.g., Adrian 2005), laser speckle velocimetry (e.g., Meynart 1983), and image correlation velocimetry (Tokumaru and Dimotakis 1995).

An important difference between these optical flow techniques and what we require for turbulent jet flow measurement is the use of time-averaging. Whereas most optical flow techniques provide the "instantaneous" or time-dependent image-velocity field, we wish to obtain an image-velocity field that is averaged over a long time interval compared to the timescales of turbulent velocity fluctuations. To obtain this time-averaged image-velocity, we apply time-averaging to a sequence of image-velocity fields obtained through a type of image-based matching. We also use two other techniques for estimating the time-averaged image-velocity field—one based on the analysis of Hovmöller diagrams in the frequency 
domain (Hovmöller 1949), and one based on temporal cross-correlation, both of which naturally incorporate time-averaging.

\subsubsection{Region-Based Matching}

Our first method for obtaining the image-velocity field is a region-based matching technique inspired by the work of Tokumaru and Dimotakis (1995). Like Tokumaru and Dimotakis (1995), we seek to track the motions of turbulent flow structures as they move across the field of view in a sequence of images. In our flows, the structures are billows that translate, rotate, and deform as they move away from the jet nozzle. We track the motion of these billows by shifting an array of subimages from one frame to find the best fit between these subimages and subimages in a subsequent frame. Unlike Tokumaru and Dimotakis (1995), we only allow subimage translation and do not account for subimage rotation or deformation because we are only interested in the velocity of the flow in the $z_{p}$-direction rather than other dynamical properties such as the vorticity.

For each image pair in a sequence, this technique results in a series of "instantaneous" image-velocity fields. We compute the mean of these fields to obtain a time-averaged image-velocity field for the time interval spanned by the image sequence.

Rather than use a simple two-dimensional cross-correlation for region matching, which tends to give faulty translation estimates when only part of a large billow is within the subimage, we compute a rootmean-square residual between two subimages using the following equation:

$$
R_{k l}=\left[\frac{\sum_{i=-I}^{I} \sum_{j=-J}^{J}\left(a_{i j}-b_{i+k, j+l}\right)^{2}}{(2 I+1)(2 J+1)}\right]^{1 / 2},
$$

where $R$ is the residual at a translation of $k$ pixels in the $x_{p}$-direction and $l$ pixels in the $z_{p}$-direction, and $a$ and $b$ define the subimage intensity at two different times. The size of subimage $a$ is given by $(2 I+1)$ in the $x_{p}$-direction and $(2 J+1)$ in the $z_{p}$-direction. The size of subimage $b$ is sufficiently larger than $a$ to allow for translations of $k$ and $l$. We over-sample $R$ by a factor of 10 using spline interpolation, and then find the position of $R$ 's minimum with respect to the zero-lag position in pixels, to obtain a single image-velocity measurement in pixels per frame.

The choice of subimage size involves a trade-off between covering an area large enough to include image intensity variations associated with flow structures, and small enough to include only flow structures that are moving at similar velocities. Because the size of flow structures generally scales with distance from 
the jet nozzle, the optimal subimage size should increase with distance from the nozzle. However in this study, rather than allow subimage sizes to vary within the image as a function of nozzle distance, we found that a subimage size of $8 \times 8$ pixels offered a good compromise between spatial resolution near the nozzle and the capture of larger flow structures farther from the nozzle. We allow these subimages to overlap by $50 \%$, to obtain image-velocity estimates on a $4 \times 4$ pixel grid.

We determined the choice of $n$, the number of image pairs to include in the mean image-velocity estimate, by examining the normalized standard error of the mean of the velocity estimates for a range of $n$. Figure 5 shows the normalized standard error of the mean as a function of $n$ at two different pixel locations along the centerline of the flow for Simulation 3. Curves are similar at other locations within the flow, and for other simulations. When $n>\sim 1000$, the normalized standard error of the mean is below $4 \%$, which is less than our estimated accuracy for measurements of the nozzle flow rate. We therefore use all the image pairs from a 30-s video clip (990 pairs) to estimate the mean image-velocity field.

\subsubsection{Hovmöller Diagram Spectral Analysis}

Our second method for obtaining the time-averaged image-velocity field is based on the spectral analysis of Hovmöller diagrams (Hovmöller 1949). Hovmöller diagrams, called "trough-and-ridge" diagrams by their inventor Ernest Hovmöller, were originally used to analyze meteorological data, and showed contours of atmospheric geopotential with the vertical axis representing latitude and the horizontal axis representing time. Many other types of data can be represented in this fashion.

We generate Hovmöller diagrams by contouring image intensity values along short vertical crosssections of 30-s video sequences, so that the vertical axis represents the spatial coordinate, and the horizontal axis represents time. We then compute the two-dimensional fast Fourier transform (FFT) of each diagram after applying a two-dimensional cosine-taper window. Figure 6 shows a typical Hovmöller diagram taken from the center of the flow in Simulation 3, and one quadrant of its corresponding FFT. The motion of flow structures in the image sequence result in a series of troughs and ridges in the Hovmöller diagram. In the frequency domain, these aligned features result in a constant ratio of the frequency, $f$, to wave number, $v$, thus creating a single ridge in the FFT. We use the location of the peaks of this ridge in the $v$-direction as the inputs to a linear regression model to find the slope of this ridge in the frequency domain. We can then convert this slope to an image-velocity.

We found that the inclusion of high-frequency information in the input to the regression model gave inconsistent results. This problem was most apparent near the plume edges or where flow was slow, probably because less high-frequency information is available in these portions of the imagery. To avoid such prob- 
lems we disregard the FFT above a cutoff frequency, which is some fraction, $c_{f}$, of the Nyquist frequency. We found empirically that setting $c_{f}$ equal to 0.5 increased the reliability of this method.

Similar to the choice of subimage size in region-based matching, the choice of cross-section height for this method involves a trade-off between covering a portion of the image large enough to include image intensity variations associated with flow structure motion, and small enough to include flow structures that are moving at similar velocities. We found that generating Hovmöller diagrams from 20-pixel vertical cross-sections provided reliable image-velocity estimates. We allow these vertical cross-sections to overlap by 19 pixels so we obtain image-velocity estimates on a $1 \times 1$ pixel grid. The velocity estimate closest to the nozzle is 10 pixels from the nozzle edge.

\subsubsection{Temporal Cross-Correlation}

Our third method for obtaining the time-averaged image-velocity field is based on the cross-correlation of image intensity values at two pixels separated in the $z_{p}$-direction. If we let $a_{i, j, k}$ represent a threedimensional pixel intensity matrix corresponding to an image sequence with $N$ frames which has been detrended at each pixel location in the time-direction, where $i, j$, and $k$ index $a$ in the $z_{p}, x_{p}$, and $t$ directions, respectively, we can calculate a cross-correlation function defined by:

$$
C_{i, j, l}(d)=\sum_{k=1}^{N-l} a_{i+d, j, k} \cdot a_{i, j, k+l}
$$

where $d$ represents the separation between pixels in the $z_{p}$-direction, and $l$ represents the lag number. We over-sample $C$ using spline interpolation to obtain fractional frame rate lag numbers, and then find the interpolated lag number $l_{\max }$ corresponding to the maximum value of $C$, and compute the image-velocity using:

$$
{\overline{u_{p}}}_{i j}=\frac{d}{l_{\max }} .
$$

Our choice of $d$ was based on a desire to have the lag numbers be as large as possible without significantly reducing the correlation coefficients in $C$. Larger lag numbers provide higher measurement accuracy as long as the signals correlate reasonably well. We found empirically that a pixel separation of $d=5$ provided a good balance between these competing factors.

This method is advantageous because it is computationally less expensive than the other two methods. It has the added advantage of emphasizing the motion of the larger and more coherent flow structures, which may reduce noise. This effect automatically accounts for the systematic differences in average 
billow size as a function of $z_{p}$. In effect, this method tunes itself to be sensitive to the larger amplitude flow features in any given region of the image sequence.

\subsection{Flow Rate Metric}

The time-averaged image-velocity field, which is a two-dimensional vector field, must be converted into a scalar metric which can be compared to the nozzle flow rate (Transform E in Figure 1). Such a metric might be calculated in a variety of ways, such as averaging the image-velocity estimates over the entire field, or averaging over some restricted area, which might be defined by some threshold associated with the image-velocity itself, some statistic associated with the image-velocity computation, or the average image intensity values in the image sequence. Another possibility is to average over some rectangular region defining a vertical cross-section, a horizontal cross-section, or some other region, possibly defined by distance from the nozzle. Other possible metrics might include averaging over the largest fraction of image-velocity estimates, or an average of the image-velocity estimates which have been weighted by some statistic associated with the image-velocity computation.

However this metric is calculated, we would like it to be related to nozzle flow rate, $W$, through some known functional relationship. When this technique is applied to pure jets, in which $\bar{u}$ scales linearly with $W$ everywhere in the flow, a linear relationship between the metric and the nozzle flow might be expected. We would expect this relationship to be zero-crossing because, disregarding noise, zero flow everywhere should give an image-velocity field of zero everywhere. When applied to pure plumes, a one-third power relationship might be expected, although a linear model could likely be assumed as long as the changes in nozzle flow rate are small. Under such an assumption the linear relationship would not in general be zerocrossing. In transitional flow, the functional relationship between $\bar{u}$ and $W$ at any position within the flow cannot be determined without knowledge of several flow properties, including the nozzle flow rate. For this reason the inclusion of image-velocity estimates from regions of transitional flow should be avoided.

In this study we calculate a flow rate metric for each of the image velocity methods by averaging together all of the values in the image-velocity field that fell within a set region of the image. This set region covered the area of the image where flow could be expected out to a distance of approximately $0.15 \mathrm{~m}$ from the nozzle opening. To establish the region where flow could be expected we used Otsu's method (Otsu 1979) to threshold the time-averaged image intensity fields for each 30-s video clip. Otsu's method seeks to minimize the intraclass variance of the pixel values above and below the threshold level, and we found empirically that one-half of the Otsu threshold level provided a good indicator of the expected flow region. We removed all of the image-velocity estimates beyond a distance of $0.15 \mathrm{~m}$ from the nozzle to remove 
the majority of estimates from the region of transitional flow. Figure 4 depicts the approximate portion of the imagery in the $z$-direction included in the flow rate metric calculation. Although the processed imagery of Simulation 1 includes some transitional flow, the included portion represents only the early part of the transition. Theory predicts that the associated bias would be small and probably insignificant.

\section{Results}

\subsection{Image-Velocity Fields}

Figure 7 shows a set of typical 30-s time-averaged image-velocity fields for Simulations 1-5, computed using the region-based matching method. The color scales have been adjusted to range from zero to one-half of the mean velocity across the nozzle, $0-W_{p} / 2$, so the results from different simulations can be compared relative to the predictions of jet theory. Significant differences between the panels represent departures from the predictions of theory for pure jets. Within the pure jet region, such differences can likely be attributed to biases associated with the image-velocity technique, or to some nonlinearity contained within the imagery. As the nozzle flow rate increases, the image-velocity estimates throughout the flow field increase. However differences between these panels, which are especially evident near the nozzle, suggest the influence of nonlinear biases.

Figure 8 shows a set of image-velocity fields computed using Hovmöller diagram spectral analysis. As in Figure 7, the color scales have been adjusted to scale with the nozzle flow rate, and in this case range from $0-W_{p}$. Overall the image-velocity values are higher with this technique, and near the nozzle these values are closer to what we might expect given the measured flow rates. However, differences between the panels—especially in the middle part of the flow field—suggest the presence of nonlinear biases.

Figure 9 shows a set of typical image-velocity fields computed using the temporal cross-correlation method. Differences between panels here are smaller than for those from the previous two techniques, suggesting the possibility of decreased bias. Image velocities near the nozzle are very close to the expected values (Table 2).

\subsection{Flow Rate Metrics}

Figure 10a shows values of the flow rate metric, $U_{p}$, computed from 1330 -s video sequences collected during each of the five simulations, plotted against the measured flow rate, $W_{p}$, for the region-based matching method. Also plotted are two linear least squares regressions—one that is forced through the origin, and one that is unconstrained in this respect. Over this range of flow rates, the relationship of $U_{p}$ to $W_{p}$ is linear 
within the error bounds of our measurements, however the large difference between the two regression lines indicates the presence of substantial bias. The non-zero-crossing regression intersects the horizontal axis at $W_{p}=-9.8$ pixels/frame. We will use this intercept value as a measure of the relative bias imparted by each technique, where a larger negative intercept suggests greater bias. The mean normalized standard deviation of these measurements is $2.7 \%$.

Figure $10 \mathrm{~b}$ shows values of the flow rate metric for Hovmöller diagram spectral analysis. The nonzero-crossing regression intersects the horizontal axis at $W_{p}=-5.6$ pixels/frame, which confirms the likely presence of nonlinear bias associated with this technique. Nonetheless the regression is linear over the range of flow rates measured, and the nonlinear bias is apparently less than that of the region-based matching technique. The mean normalized standard deviation is $1.8 \%$.

Figure 10c shows the values of the flow rate metric for temporal cross-correlation. These two regressions are statistically distinct, indicating the continued presence of some nonlinear bias. However, the non-zero-crossing regression intersects the horizontal axis at $W_{p}=-2.6$ pixels/frame, suggesting that this method is the least biased of the three. The mean normalized standard deviation is $2.6 \%$.

\section{Discussion}

\subsection{Sources of Bias}

All three OPV techniques provided approximately linear relationships between nozzle flow rate and the flow rate metric over the range of flow rates examined. None of the techniques provided the zero-crossing relationship that we would expect for a metric derived from imagery of a pure jet. A non-zero-crossing regression implies the existence of some nonlinear bias, which can be thought of as real, (i.e., associated with the flow), or artificial (i.e., associated with the computational technique). Flow-related bias is likely to affect our results equally regardless of the image-velocity technique because the bias is contained within the imagery of the flow.

Region-based matching has the largest bias. One potential source of bias is related to noisy imagevelocity estimates. A noisy image-velocity estimate is generated when the residual, $R$, is computed from two subimages that do not contain flow features that can be correlated between frames. This might occur when there are no flow features at all in one or both subimages, or when the flow features are changing shape rapidly. The result is a residual minimum that occurs at a random position, which adds noise to the image-velocity estimates that has a velocity mean of zero. This would drive image-velocity estimates down 
overall, and might explain why the flow rate metrics derived using this method are lower overall. Because such noise might increase with increasing flow rate, this noise is also a potential source of nonlinear bias.

One way to address this problem might be to characterize the quality of each image-velocity estimate based on some aspect of the residual function. This could be accomplished by using an F-test to estimate confidence limits for the minimum of $R$ (Draper and Smith 1981; Wilcock and Toomey 1991), or by analyzing the curvature of $R$ near the residual minimum, similar to the technique discussed by Anandan (1989). We made some preliminary efforts to implement such techniques, but were unable to establish a characterization scheme that significantly reduced the noise. It also became clear that the region-based matching technique was computationally expensive relative to the other techniques, and the employment of sophisticated noise reduction schemes would likely exacerbate this issue.

Hovmöller diagram spectral analysis has the second largest bias. A potential source of bias here is related to the frequency-based cutoff in the Hovmöller FFT regression. Applying a frequency cutoff when fitting a regression curve to the peaks on the FFT effectively results in the low-pass filtering of the data in the spatial domain. The wavelengths filtered, however, are a function of image-velocity. For a given cutoff value, higher image-velocities result in longer wavelength features being filtered when we apply the cutoff to the Hovmöller FFT data. This may bias our result if different size features have systematically different velocities. One potential way to address this issue is to change the frequency cutoff as a function of imagevelocity. This would require the implementation of some iterative scheme, whereby the image-velocity was first estimated and then refined as the frequency cutoff was changed.

Temporal cross-correlation has the lowest bias. Unlike the previous two techniques, we can think of no nonlinear bias that might be associated with this image-velocity method. Rather, we suspect that this image-velocity technique is providing an accurate measure of the time-averaged velocities of flow structures in the image sequence, with flow-related bias contained in the imagery.

One potential source of flow-related bias is related to the viewable depth, recalling that the viewable depth is the time-averaged distance the imaging system "sees" into the flow. It is possible that the viewable depth is greater when the flow is slower. Turbulence theory predicts that the smallest scales of turbulence will become smaller with increasing Reynolds number (Dimotakis et al 1983). This suggests that "holes" in the flow, which allow the observation of deeper sections of the plume, might be shallower at higher Reynolds numbers. One way to estimate the magnitude of this effect is to examine the structure of the edges of the flow as a function of nozzle flow rate. We use the variance of the edge position, normalized for plume width, as a proxy for viewable depth. Figure 11 helps illustrate this procedure. For each 30$\mathrm{s}$ video sequence, we compute the mean and standard deviation of the flow edge, which is defined by a constant image intensity level. We found a level of 40 (on a scale of 0-255) to provide a reasonable 
estimate of the edge of the flow. Because the standard deviation increases with height, as does plume width, we normalize the standard deviation by the plume's half-width at each distance from the nozzle, and average all of the values together to obtain a single measurement of the edge standard deviation for each 30-s sequence.

Figure 12 shows a plot of these values for 13 30-s sequences collected during each of five simulations. The plume edge variance decreases with increasing nozzle flow rate by approximately $20 \%$ over the range of flow rates we consider. A back-of-the-envelope calculation, appealing to the theoretical distribution of mean velocities in the flow, shows that a decrease in viewable depth of $20 \%$ may produce a decrease in the image-velocity of about $5-10 \%$. This is not sufficient to entirely explain the non-zero-crossing regression obtained using the region-based matching method or the Hovmöller diagram method. However, it may be enough to explain the smaller bias observed using the temporal cross-correlation method.

\subsection{Implications for Seafloor Systems}

Our study inspires confidence that relative flow rates can be obtained in real black smoker systems using OPV. The best image analysis technique evaluated in this study is the temporal cross-correlation method, which is computationally least expensive, and works well without much user intervention. Further, there are no obvious biases associated with this technique, as there are for the other two techniques.

OPV will be most sensitive to changes in flow rate when pure jets are imaged. OPV will work when pure plumes are imaged, but its sensitivity will be reduced. OPV will not work well when transitional flows are imaged. In this case, increasing nozzle flow rate will result in an increasing flow rate metric, but the effect of changing Morton length scales will render even relative flow rate changes ambiguous. Different relationships between the nozzle flow rate and $\bar{u}$ in differing parts of the imaged flow will further reduce this technique's effectiveness in transitional flows. Care should be taken to minimize the imaging of transitional flow when utilizing OPV.

Figure 13 shows the theoretical transition zones for two black smokers as a function of distance from the nozzle, $z$, and mean nozzle velocity, $W$. For a given nozzle velocity, hotter and smaller black smokers will transition to plume-like behavior closer to the nozzle and over a shorter distance than cooler and larger black smokers. Here we show two "end-member" black smokers—one that is hot and small, and one that is cool and large. Most typical black smokers will exhibit pure plume-like behavior within about $1 \mathrm{~m}$ of the nozzle. This may extend to $2 \mathrm{~m}$ for large and relatively cool black smokers that are flowing very fast. The pure jet region can be very small, from just a few centimeters up to about $0.5 \mathrm{~m}$. 
While theory suggests that higher sensitivities are obtainable in the pure jet region, imaging the plume region may be easier because in this part of the flow billows are relatively larger, move more slowly, and deform less from frame to frame. For OPV, these separate advantages are offsetting to some degree. However, by increasing the video resolution and frame rate, the likelihood of aliasing in the jet region can be reduced. In this study we found that standard frame rate video was just barely capable of providing flow rate information near the jet nozzle, and higher frame rates would have been beneficial had the camera been able to support them. We would thus recommend that any future seafloor OPV system be designed to focus on the pure jet region and include a high-speed imaging system to resolve the smaller and faster-moving flow structures found near the nozzle.

In real black smoker systems the viewable depth issue may not be as much of a problem as in our simulations. The Reynolds numbers of black smokers are much higher than those of our laboratory flows, and "deep" views into real black smokers may occur less often. Also, real black smokers may be more opaque than our simulated flows because near the nozzle they increase their opacity during mixing through mineral precipitation. In our simulations opacity decreased with mixing, which may have contributed to the viewable depth issue.

The precision values we calculate for the 30-s image sequences suggest that under ideal conditions the measurement resolution in a real system could be on the order of a few percent. This might be improved to some extent because in real systems we would seek to measure variability with timescales that are tidal and longer, allowing multiple 30-s measurements to be averaged into a single measurement. This level of precision is likely sufficient to resolve flow variability that is expected in real systems, however there are other complicating factors that need to be considered. These include potential changes to the flow associated with ocean currents, changes to the nozzle shape and size associated with mineral precipitation, and changes to the instrumentation caused by biofouling on the camera and lights. It is difficult to quantify the degree to which these factors will affect OPV, but the laboratory precision estimates cannot be used to infer flow rate changes without thinking about whether the changes are real.

In regard to the analysis of historical video, our results suggest that OPV can potentially be used to extract flow rates from these data. The historical video data are contained on analog tapes in the NTSC format, and they show effluent flow from several different vents in the Main Endeavour field on the Juan de Fuca Ridge (McDuff and Delaney 1995). Short video segments were collected at periodic intervals over weeks and months in the late 1980s and early 1990s. These data likely have frame rates that will be sufficient for OPV analysis. However, the pure jet region is imaged in a small portion of the image frames, so spatial resolution may be an issue, but we cannot be certain until we begin our analysis. 


\section{Conclusions}

In this paper we described laboratory experiments used to obtain video image sequences of flow-rate controlled turbulent buoyant jets for analysis with an image-based time-averaging flow measurement technique. We then discussed the results of our analysis, which we carried out using three different imagevelocity techniques. The principal conclusions of this study are as follows:

1. The three image-velocity techniques used in this study provided approximately linear relationships between the flow rate metric and the nozzle flow rate over the range of nozzle flow rates simulated in our laboratory experiments.

2. None of these techniques provided a zero-crossing relationship, suggesting the presence of one or more nonlinear biases, which could be artificial (i.e., related to our technique), or real (i.e., related to some aspect of the flow).

3. Artificial nonlinear bias could be related to a noise component that increases with nozzle flow rate, as in the region-based matching technique, or filtering which changes as a function of nozzle flow rate, as in the Hovmöller diagram technique.

4. Real nonlinear bias may be related to the viewable depth of the flow being dependent upon flow rate.

5. Region-based matching incorporated more noise into the time-averaged image-velocity estimate. This effect was strongest near the nozzle. The computational expense of this technique and the noise issue probably preclude the use of our implementation of region-based matching in OPV.

6. Hovmöller diagram spectral analysis worked reasonably well, but velocity-dependent filtering may have biased the flow rate estimates. Some type of iterative adaptive filtering may alleviate this issue.

7. Temporal cross-correlation produced excellent image-velocity results. This method still produced a non-zero-crossing relationship between the flow rate metric and the nozzle flow rate, but this may be a real effect (i.e. related to the flow rather than the computational method). This is the most computationally efficient technique, and the preferred image-velocity technique for use in OPV.

8. Video image analysis has the potential to provide relative flow rate information in real systems. Highspeed high-resolution camera systems are recommended for use in the pure jet region of black smoker flow. Care should be taken to avoid imaging or analyzing transitional flows to avoid introducing ambiguity into the flow rate estimates. 


\section{Electronic Supplementary Material}

Supplementary material that accompanies this work is available online. The captions for this material are presented below.

Movie 1: Movie showing thirty seconds of video data from Simulation 1, in which the flow exited the nozzle with an average velocity of $0.16 \mathrm{~m} / \mathrm{s}$.

Movie 2: Movie showing thirty seconds of video data from Simulation 2, in which the flow exited the nozzle with an average velocity of $0.21 \mathrm{~m} / \mathrm{s}$.

Movie 3: Movie showing thirty seconds of video data from Simulation 3, in which the flow exited the nozzle with an average velocity of $0.27 \mathrm{~m} / \mathrm{s}$.

Movie 4: Movie showing thirty seconds of video data from Simulation 4, in which the flow exited the nozzle with an average velocity of $0.34 \mathrm{~m} / \mathrm{s}$.

Movie 5: Movie showing thirty seconds of video data from Simulation 5, in which the flow exited the nozzle with an average velocity of $0.37 \mathrm{~m} / \mathrm{s}$.

Acknowledgements We thank John Kumph, Eric Lindahl, and John Mickett for many helpful discussions about this work, and we thank Evelyn Lessard for the use of camera equipment. This manuscript benefited greatly from the thorough reviews of two anonymous reviewers. Support for this study was provided by the National Science Foundation under grant OCE0623285, by the W. M. Keck Foundation through a grant to the University of Washington, and by John Delaney via the Jerome M. Paros Endowed Chair in Sensor Networks.

\section{References}

Adelson EH, Bergen JR (1985) Spatiotemporal energy models for the perception of motion. J Opt Soc Am A 2(2):284-299 Adrian RJ (2005) Twenty years of particle image velocimetry. Exp Fluid 39:159-169, DOI 10.1007/s00348-005-0991-7

Alt JC (1995) Subseafloor processes in mid-ocean ridge hydrothermal systems. In: Humphris SE, Zierenberg RA, Mullineaux LS, Thomson RE (eds) Seafloor Hydrothermal Systems: Physical, Chemical, Biological, and Geological Interactions, Geophysical Monograph 91, American Geophysical Union, Washington DC, pp 85-114

Anandan P (1989) A computational framework and an algorithm for the measurement of visual motion. Int J Comput Vis $2: 283-310$

Beauchemin SS, Barron JL (1995) The computation of optical flow. ACM Comput Surv 27(3):433-467

Bischoff JL, Rosenbauer RJ (1985) An empirical equation of state for hydrothermal seawater (3.2 percent NaCl). Am J Sci 285(8):725-763

Butterfield DA, Jonasson IR, Massoth GJ, Feely RA, Roe KK, Embley RW, Holden JF, McDuff RE, Lilley MD, Delaney JR (1997) Seafloor eruptions and evolution of hydrothermal fluid chemistry. Phil Trans Roy Soc Lond A 355:369-386

Converse DR, Holland HD, Edmond JM (1984) Flow rates in the axial hot springs of the East Pacific Rise (21 $\left.{ }^{\circ} \mathrm{N}\right)$ : Implications for the heat budget and the formation of massive sulfide deposits. Earth Planet Sci Lett 69:159-175 
Coupland JM (2000) Laser Doppler and pulsed laser velocimetry in fluid mechanics. In: Rastogi PK (ed) Photomechanics, Topics in Applied Physics, Springer-Verlag, Berlin

Crone TJ, Wilcock WSD (2005) Modeling the effects of tidal loading on mid-ocean ridge hydrothermal systems. Geochem Geophys Geosyst 6:Q07001, DOI 10.1029/2004GC000905

Crone TJ, Wilcock WSD, Barclay AH, Parsons JD (2006) The sound generated by mid-ocean ridge black smoker hydrothermal vents. PLoS ONE 1:e133, DOI 10.1371/journal.pone.0000133

Davis E, Becker K, Dziak R, Cassidy J, Wang K, Lilley M (2004) Hydrological response to a seafloor spreading episode on the Juan de Fuca Ridge. Nature 430:335-338, DOI 10.1038/nature02755

Delaney JR, Robigou V, McDuff RE (1992) Geology of a vigorous hydrothermal system on the Endeavour segment, Juan de Fuca Ridge. J Geophys Res 97(B13):19,663-19,682

Delaney JR, Kelley DS, Lilley MD, Butterfield DA, Baross JA, Wilcock WSD, Embley RW, Summit M (1998) The quantum event of oceanic crustal accretion: Impacts of diking at mid-ocean ridges. Science 281:222-230

Delaney JR, Heath GR, Howe B, Chave AD, Kirkham H (2000) NEPTUNE: Real-time ocean and earth sciences at the scale of a tectonic plate. Oceanography 13(2):71-79

Dimotakis PE, Miake-Lye RC, Papantoniou DA (1983) Structure and dynamics of round turbulent jets. Phys Fluids 26(11):3185-3192

Draper NR, Smith H (1981) Applied Regression Analysis. Wiley, New York

Elderfield H, Schultz A (1996) Mid-ocean ridge hydrothermal fluxes and the chemical composition of the ocean. Annu Rev Earth Planet Sci 24:191-224

Harris FJ (1978) On the use of windows for harmonic analysis with the discrete Fourier transform. Proc IEEE 66:51-83

Holzbecher EO (1998) Modeling Density-Driven Flow in Porous Media. Springer-Verlag, Berlin

Horn BKP, Schunck BG (1981) Determining optical flow. Artif Intell 17:185-203

Hovmöller E (1949) The trough-and-ridge diagram. Tellus 1(2):62-66

Johnson HP, Hutnak M, Dziak RP, Fox CG, Urcuyo I, Cowen JP, Nabelek J, Fisher C (2000) Earthquake-induced changes in a hydrothermal system on the Juan de Fuca mid-ocean ridge. Nature 407:174-177

Jupp TE (2000) Fluid flow processes at mid-ocean ridge hydrothermal systems. PhD thesis, Pembroke College, University of Cambridge

Kelley DS, Baross JA, Delaney JR (2002) Volcanoes, fluids, and life at mid-ocean ridge spreading centers. Annu Rev Earth Planet Sci 30:385-491, DOI 10.1146/annurev.earth.30.091201.141331

Kraus NC, Lohrmann A, Cabrera R (1994) New acoustic meter for measuring 3D laboratory flows. J Hydraul Eng 120(3):406-412

Larson BI, Olson EJ, Lilley MD (2007) In-situ measurement of dissolved chloride in high temperature hydrothermal fluids. Geochim Cosmochim Acta 71:2510-2523, DOI 10.1016/j.gca.2007.02.013

List EJ (1982) Turbulent jets and plumes. Annu Rev Fluid Mech 14:189-212

McDuff RE (1995) Physical dynamics of deep-sea hydrothermal plumes. In: Humphris SE, Zierenberg RA, Mullineaux LS, Thomson RE (eds) Seafloor Hydrothermal Systems: Physical, Chemical, Biological, and Geological Interactions, Geophysical Monograph 91, American Geophysical Union, Washington DC, pp 357-368

McDuff RE, Delaney JR (1995) Periodic variability in fluid temperature at a seafloor hydrothermal vent. Eos Trans AGU 76(46), Fall Meet. Supl., F710

Meynart R (1983) Speckle velocimetry study of vortex pairing in a low-Re unexcited jet. Phys Fluids 26:2074-2079 
Morton BR, Taylor G, Turner JS (1956) Turbulent gravitational convection from maintained and instantaneous sources. Proc Roy Soc Lond Math Phys Sci 234(1196):1-23

Otsu N (1979) A threshold selection method from gray-level histograms. IEEE Trans Syst Man Cybern 9:62-66

Papanicolaou PN, List EJ (1988) Investigations of round vertical turbulent bouyant jets. J Fluid Mech 195:341-391

Pruis MJ, Johnson HP (2004) Tapping into the sub-seafloor: examining diffuse flow and temperature from an active seamount on the Juan de Fuca Ridge. Earth Planet Sci Lett 217:379-388, DOI 10.1016/S0012-821X(03)00607-1

Rodi W (ed) (1982) Turbulent Buoyant Jets and Plumes, HMT-The Science \& Applications of Heat and Mass Transfer, vol 6. Pergamon, New York

Ruden P (1933) Turbulente Ausbreitungsvorgänge im Freistrahl. Die Naturwissenschaften 21:375-378

Schultz A, Dickson P, Elderfield H (1996) Temporal variations in diffuse hydrothermal flow at TAG. Geophys Res Lett 23(23):3471-3474

Shabbir A, George WK (1994) Experiments on a round turbulent buoyant plume. J Fluid Mech 275:1-32

Spiess FN, Macdonald KC, Atwater T, Ballard R, Carranza A, Cordoba D, Cox C, Diaz Garcia VM, Francheteau J, Guerrero J, Hawkins J, Haymon R, Hessler R, Juteau T, Kastner M, Larson R, Luyendyk B, Macdougall JD, Miller S, Normark W, Orcutt J, Rangin C (1980) East Pacific Rise: Hot springs and geophysical experiments. Science 207(4438):1421-1433

Tokumaru PT, Dimotakis PE (1995) Image correlation velocimetry. Exp Fluid 19:1-15

Tolstoy M, Vernon FL, Orcutt JA, Wyatt FK (2002) Breathing of the seafloor: Tidal correlations of seismicity at Axial volcano. Geology 30(6):503-506

Turner JS (1986) Turbulent entrainment: The development of the entrainment assumption, and its application to geophysical flows. J Fluid Mech 173:431-471

Wilcock WSD, Fisher AT (2004) Geophysical constraints on the sub-seafloor environment near mid-ocean ridges. In: Wilcock WSD, DeLong EF, Kelley DS, Baross JA, Cary SC (eds) The Subseafloor Biosphere at Mid-Ocean Ridges, Geophysical Monograph 144, American Geophysical Union, Washington DC, pp 51-74

Wilcock WSD, Toomey DR (1991) Estimating hypocentral uncertainties for marine microearthquake surveys: A comparison of the generalized inverse and grid search methods. Mar Geophys Res 13:161-171 
Table 1 Flow rate measurements for laboratory simulations. $\Delta t_{10}$ is the time for the source fluid tank level to drop by 10 liters. $Q_{i}$ is the corresponding flow rate through the nozzle, and $Q_{m}$ is the mean flow rate for each simulation. $S$ is the bias-corrected standard deviation normalized by $Q_{m}$ and multiplied by 100 .

\begin{tabular}{|c|c|c|c|c|}
\hline Simulation & $\Delta t_{10}(\mathrm{~s})$ & $Q_{i}(1 / \mathrm{s})$ & $Q_{m}(1 / \mathrm{s})$ & $S(\%)$ \\
\hline \multirow{3}{*}{1} & 256 & 0.039 & \multirow{3}{*}{0.041} & \multirow{3}{*}{4.0} \\
\hline & 243 & 0.041 & & \\
\hline & 237 & 0.042 & & \\
\hline \multirow{4}{*}{2} & 176 & 0.057 & \multirow{4}{*}{0.054} & \multirow{4}{*}{5.3} \\
\hline & 197 & 0.051 & & \\
\hline & 192 & 0.052 & & \\
\hline & 180 & 0.056 & & \\
\hline \multirow{4}{*}{3} & 146 & 0.068 & \multirow{4}{*}{0.068} & \multirow{4}{*}{1.7} \\
\hline & 151 & 0.066 & & \\
\hline & 146 & 0.068 & & \\
\hline & 146 & 0.068 & & \\
\hline \multirow{5}{*}{4} & 115 & 0.087 & \multirow{5}{*}{0.086} & \multirow{5}{*}{5.4} \\
\hline & 118 & 0.085 & & \\
\hline & 122 & 0.082 & & \\
\hline & 106 & 0.094 & & \\
\hline & 120 & 0.083 & & \\
\hline \multirow{5}{*}{5} & 109 & 0.092 & \multirow{5}{*}{0.094} & \multirow{5}{*}{3.1} \\
\hline & 109 & 0.092 & & \\
\hline & 103 & 0.097 & & \\
\hline & 102 & 0.098 & & \\
\hline & 107 & 0.093 & & \\
\hline
\end{tabular}

Table 2 Derived flow rate information for laboratory simulations.

\begin{tabular}{cccc}
\hline Simulation & $W(\mathrm{~m} / \mathrm{s})$ & $W_{p}$ (pixels/frame) & $R e$ \\
\hline 1 & 0.16 & 4.2 & 2900 \\
2 & 0.21 & 5.5 & 3800 \\
3 & 0.27 & 7.0 & 4900 \\
4 & 0.34 & 8.8 & 6200 \\
5 & 0.37 & 9.6 & 6700 \\
\hline
\end{tabular}




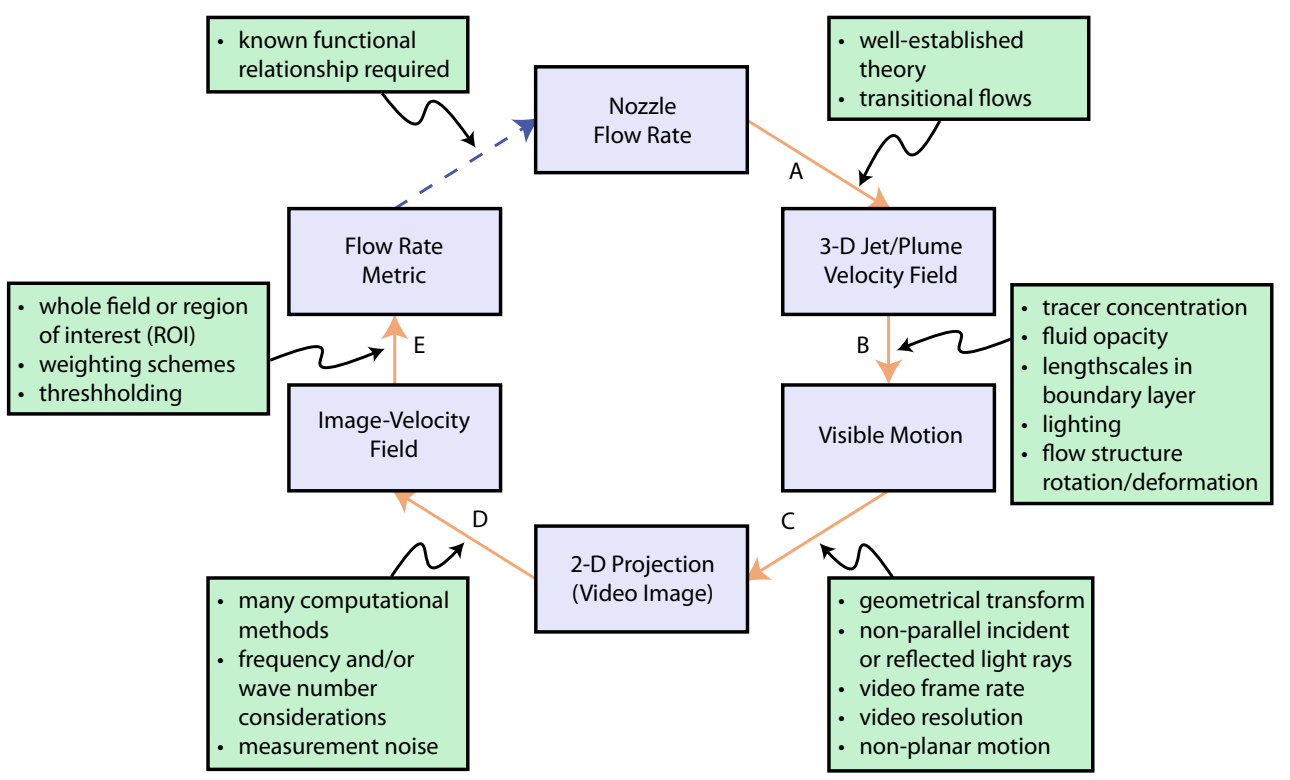

Fig. 1 Conceptual flow diagram illustrating the stages of image-based jet flow measurement. Blue boxes depict the various stages of the process, from "Nozzle Flow Rate", which is the quantity desired, to "Flow Rate Metric", which is the estimate of that quantity. Orange arrows represent the various physical, optical, and computational transforms between stages of this process, which are labeled and referred to in the text as Transforms A-E. Green boxes show the issues and complicating factors associated with the transform functions which we discuss at length in the text. The blue dashed arrow represents the relationship between the flow rate metric and the nozzle flow rate, which should have a known functional form. 


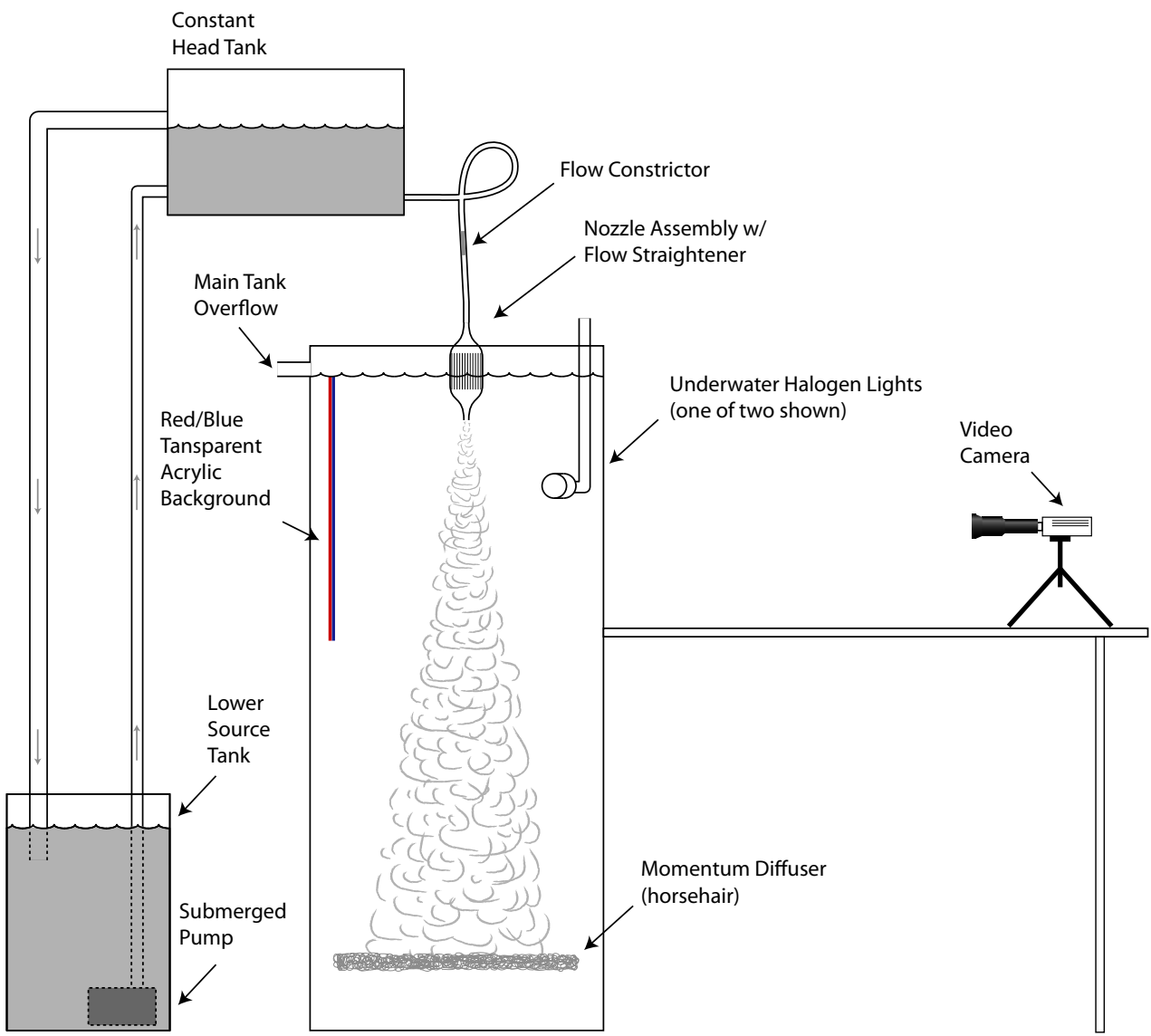

Fig. 2 Schematic of simulated black smoker jet apparatus. The major components are drawn approximately to scale. The main tank measured $2.1 \mathrm{~m}$ tall and was $0.9 \mathrm{~m}$ square. A submerged pump supplied jet fluid from the source tank to a constanthead tank. A momentum diffuser near the bottom of the tank reduced the tendency for the jet fluid to return to the upper part of the main tank. 


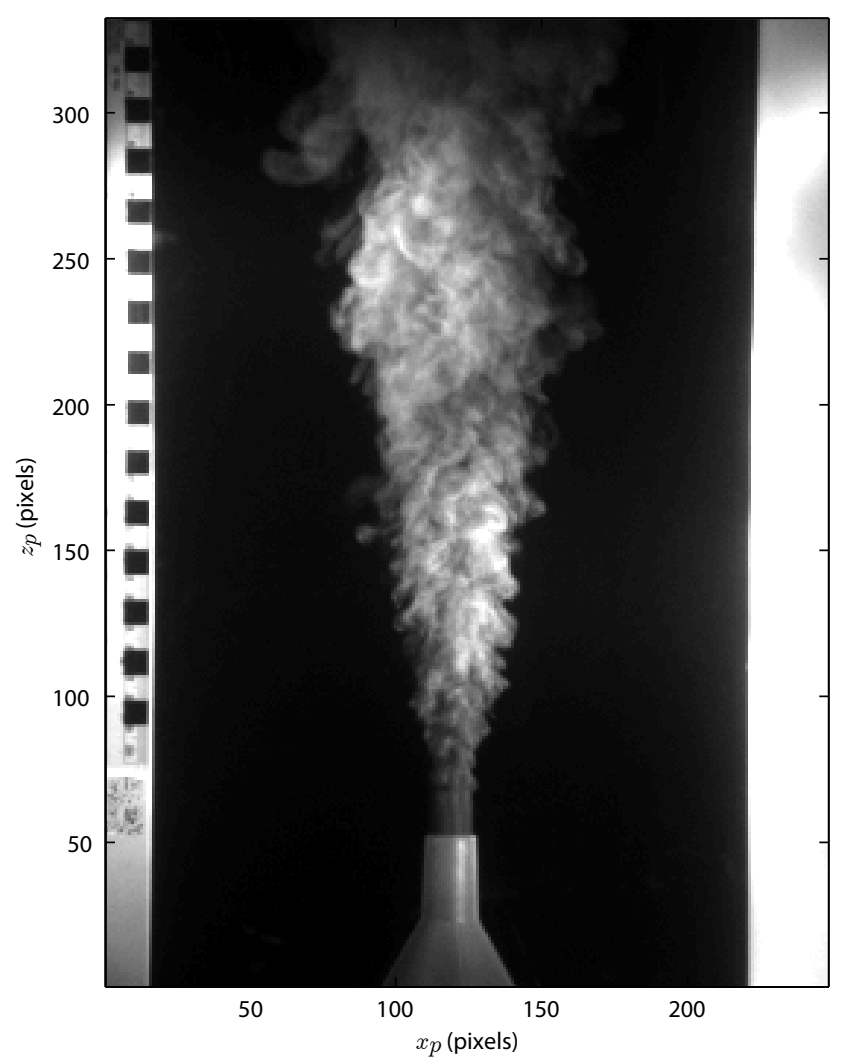

Fig. 3 Typical video image frame from a black smoker simulation. Although the experiments were run with the jets flowing downward, in this paper we rotate all of the jet and image-velocity plots so the $z$-axis is positive upward. The scale bar on the left-hand side is composed of black and white blocks measuring $0.01 \mathrm{~m}$ square. 


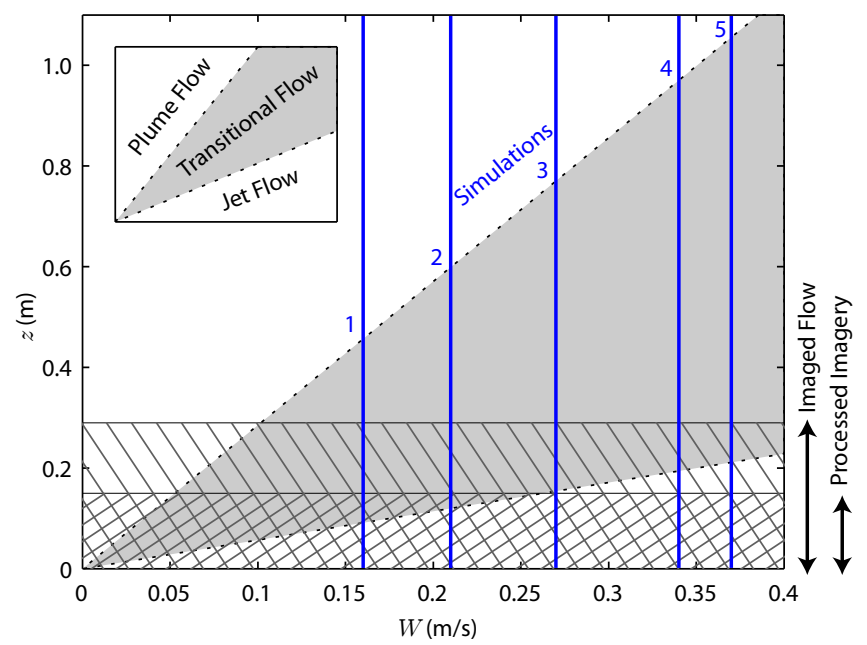

Fig. 4 Relative estimated distances over which the simulated flows transition from pure jets to pure plumes. The gray shaded area represents transitional flow, separating pure jets below from pure plumes above. The hatched area depicts the amount of the flow imaged, in the $z$-direction, which measured approximately $0.3 \mathrm{~m}$. The cross-hatched area depicts the amount of the imaged flow that was included in the flow rate metric, in the $z$-direction. Our goal was to include some transitional flow in the imagery, as would be expected in the historical data, but we included little transitional flow in our analyses.

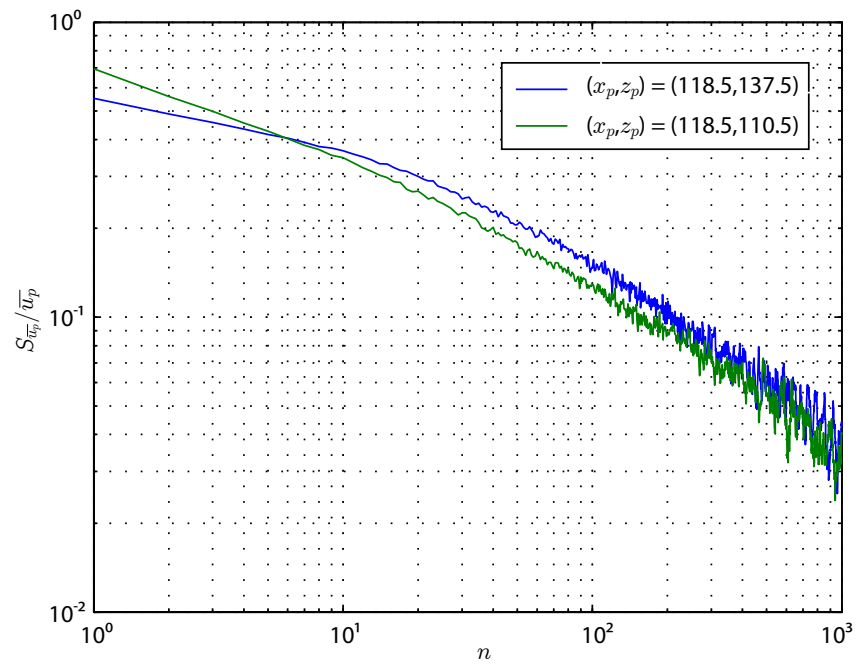

Fig. 5 Normalized standard error of the mean of the mean image-velocity, $\overline{u_{p}}$, as a function of $n$, the number of instantaneous image-velocity estimates included in the mean. The green and blue lines show the quantity computed at two locations along the centerline of the flow, at approximately 0.1 and $0.2 \mathrm{~m}$ from the nozzle, during Simulation 3. The variability of these curves increases as $n$ increases because the population size is fixed, so the number of sample means used in the standard error calculation decreases. When $n$ is $\sim 1000$ the normalized standard error of the mean is below $4 \%$. 


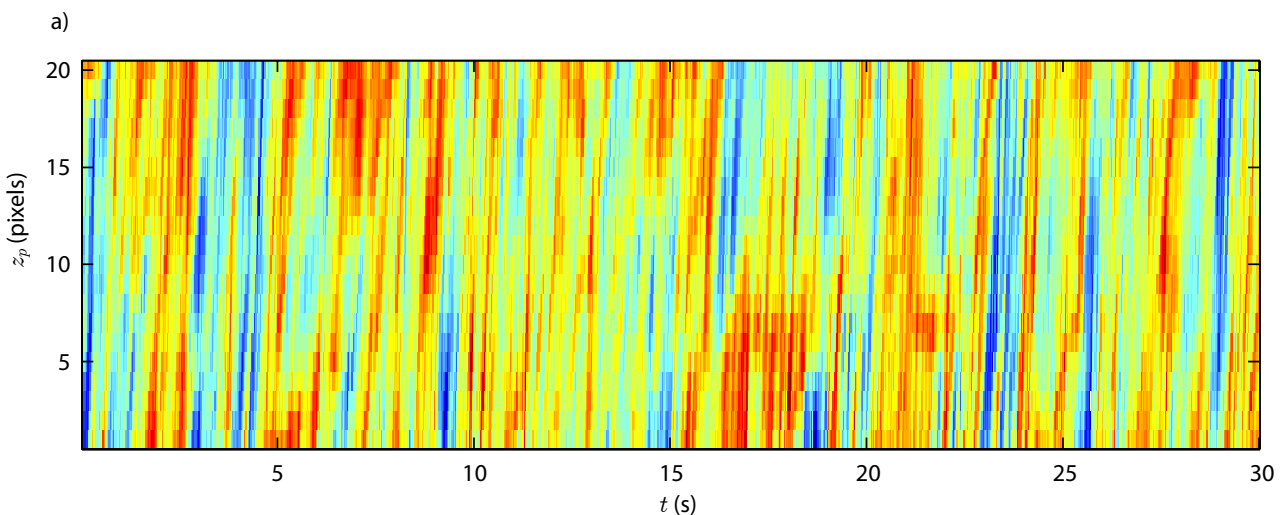

b)

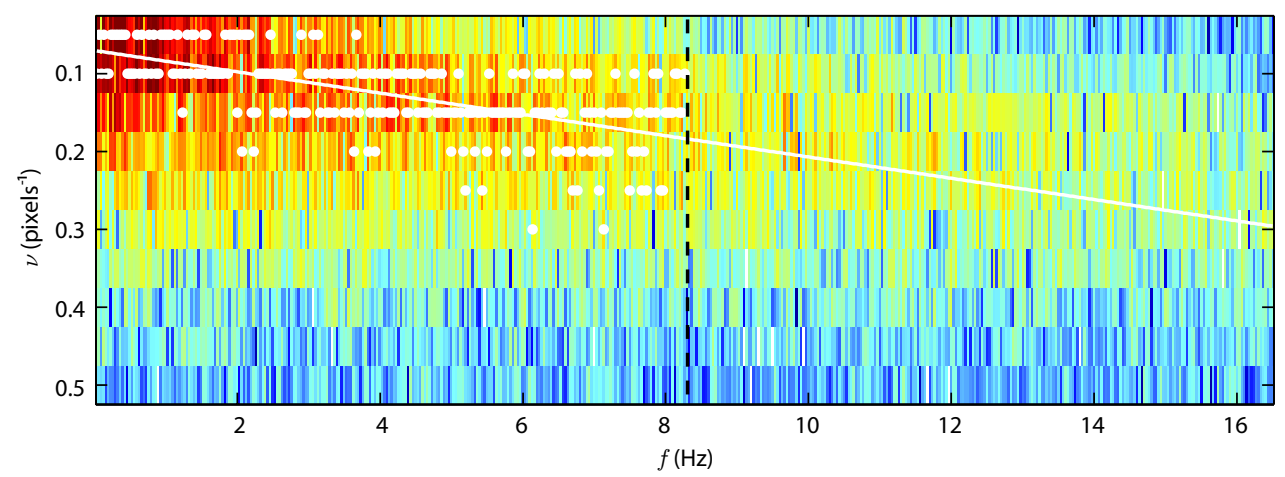

Fig. 6 Typical Hovmöller diagram with least squares plane subtracted (a), and one quadrant of the corresponding twodimensional FFT (b). Horizontal axes have been converted to time (s) and frequency (Hz) using a frame rate of 33 frames per second. The Hovmöller diagram is windowed with a two-dimensional Tukey (cosine-taper) window (Harris 1978) before the FFT is computed. The white line is a least squares fit to the peaks of the FFT in the $v$-direction, shown by the white dots $\left(R^{2}=0.40\right)$. We only included peaks up to a cutoff frequency shown by the black dashed line. The slope of the white line is converted to the image-velocity associated with this Hovmöller diagram. 
$\mathrm{px} / \mathrm{s}$
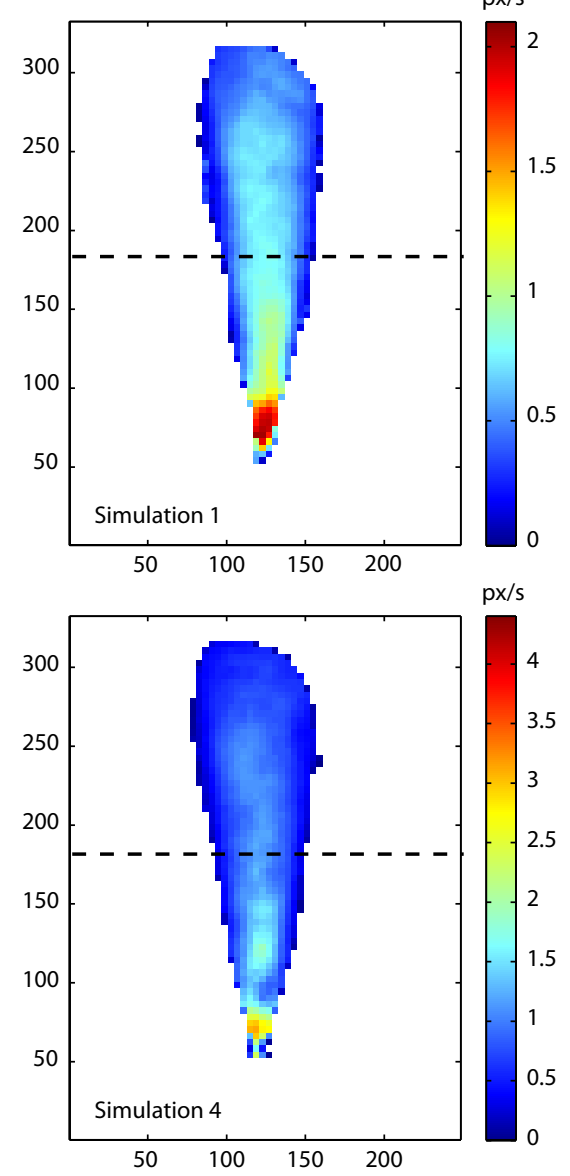

$\mathrm{px} / \mathrm{s}$

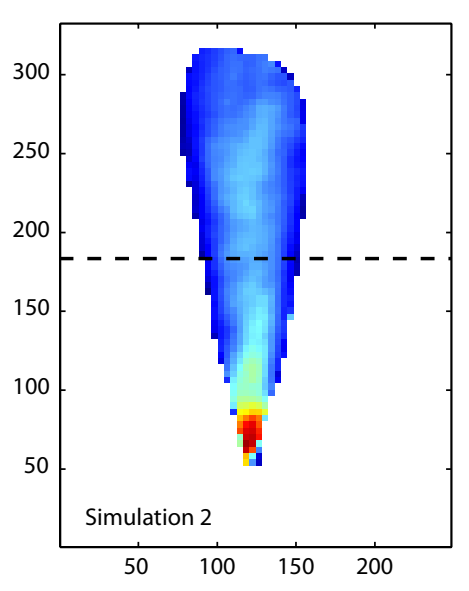

25

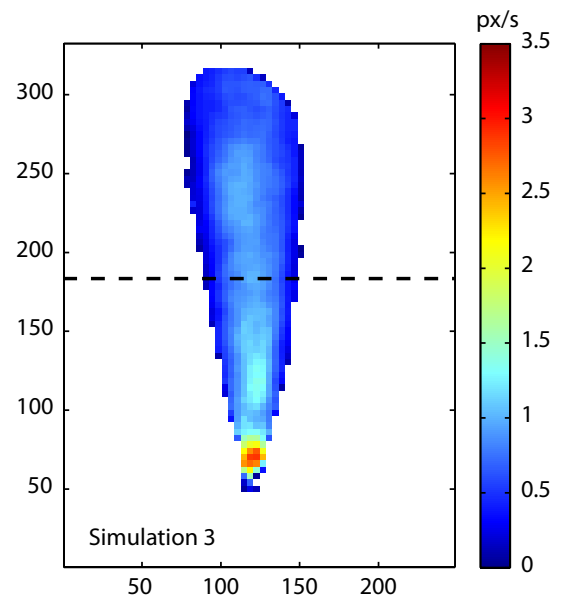

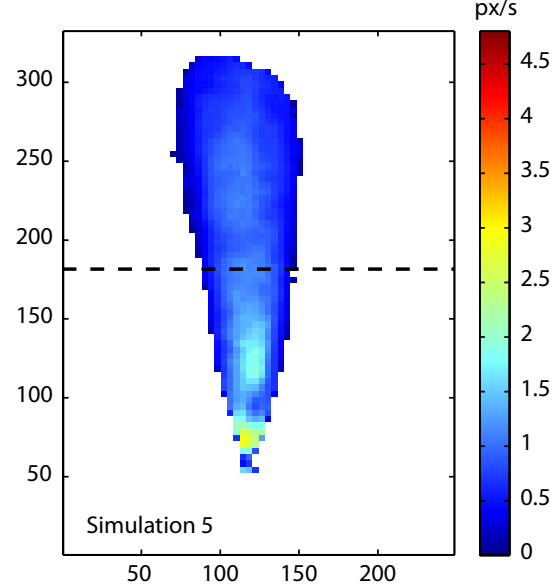

Fig. 7 Typical 30-s time-averaged image-velocity fields computed using region-based matching. Color-filled contours of image-velocity are shown in pixels per frame with the color scale adjusted from zero to one-half of the mean measured velocity across the nozzle, $W_{p}$ (See Table 2 ). The parts of the image-velocity field colored white have been discarded using Otsu thresholding as described in the text. The image-velocity field below the black dashed line is included in the flow rate metric. Because the color scale is scaled with $W_{p}$, significant differences between the panels represent departures from theoretical predictions of pure jet behavior. 

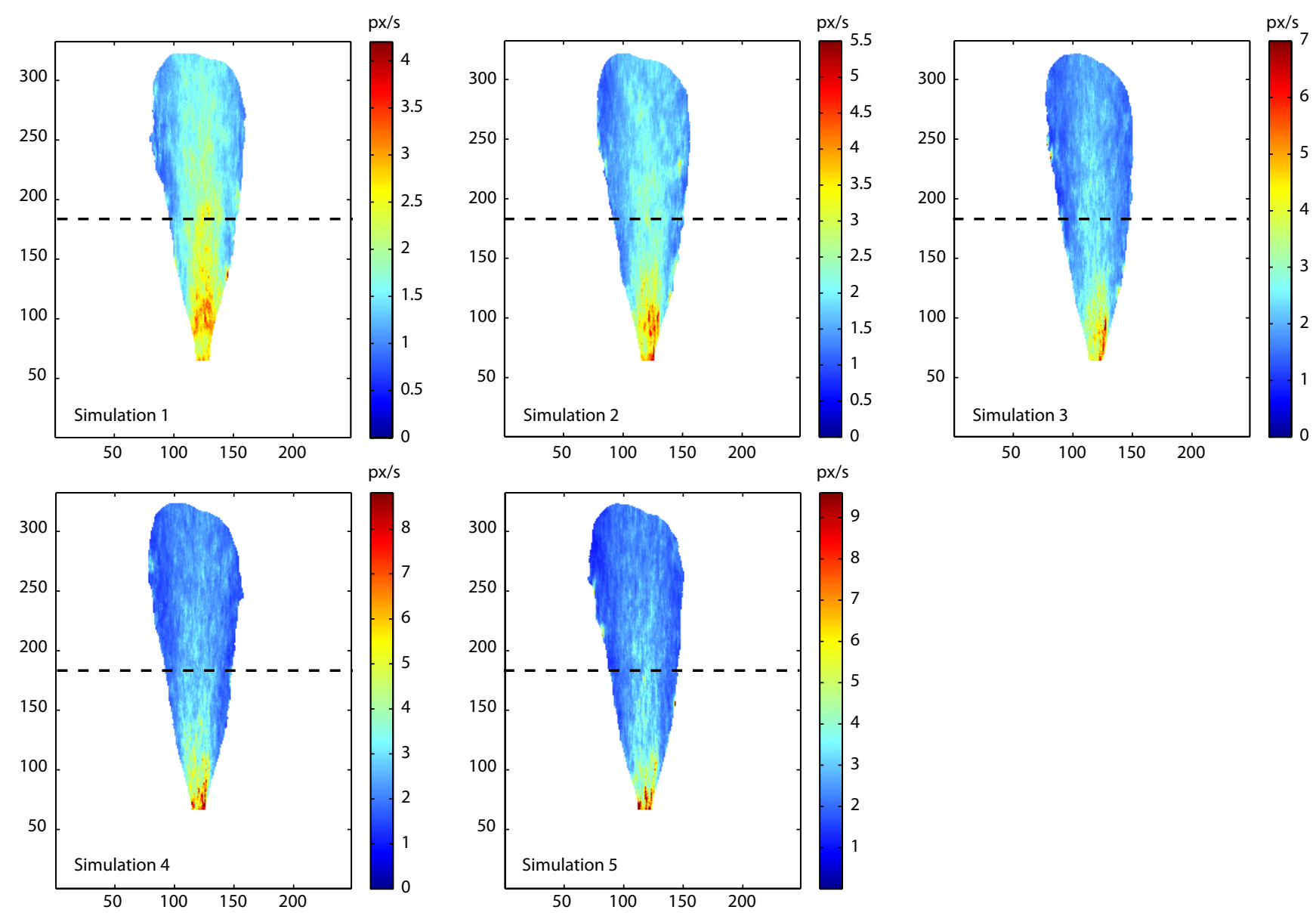

Fig. 8 Same as Figure 7 except these image-velocity fields were computed using Hovmöller diagram spectral analysis. Because the image-velocities are higher overall, the color scale is adjusted from zero to the mean measured velocity across the nozzle, $W_{p}$ (See Table 2). 

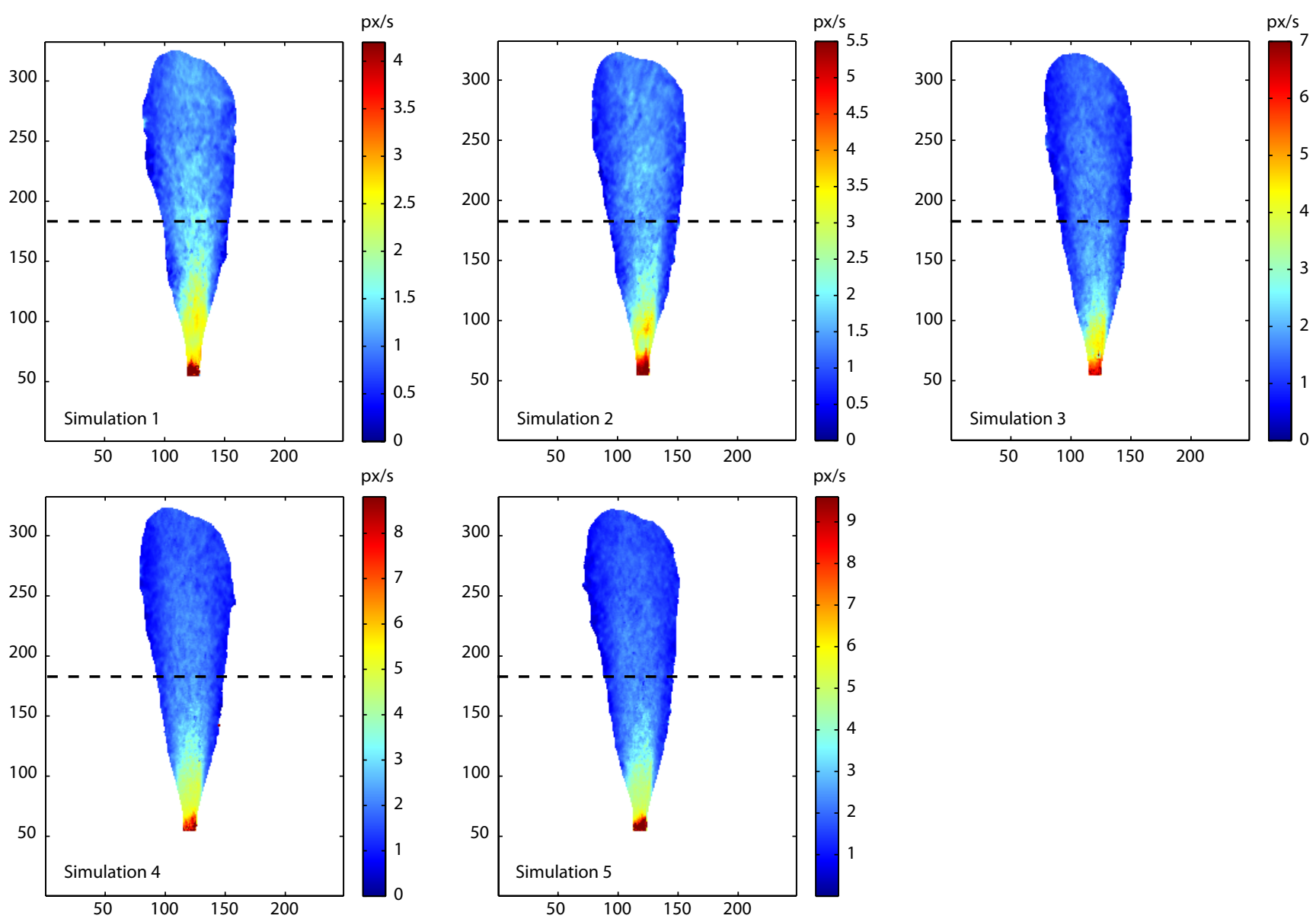

Fig. 9 Same as Figure 8 except these image-velocity fields were computed using temporal cross-correlation. Because the image-velocities are higher overall, the color scale is adjusted from zero to the mean measured velocity across the nozzle, $W_{p}$ (See Table 2). 

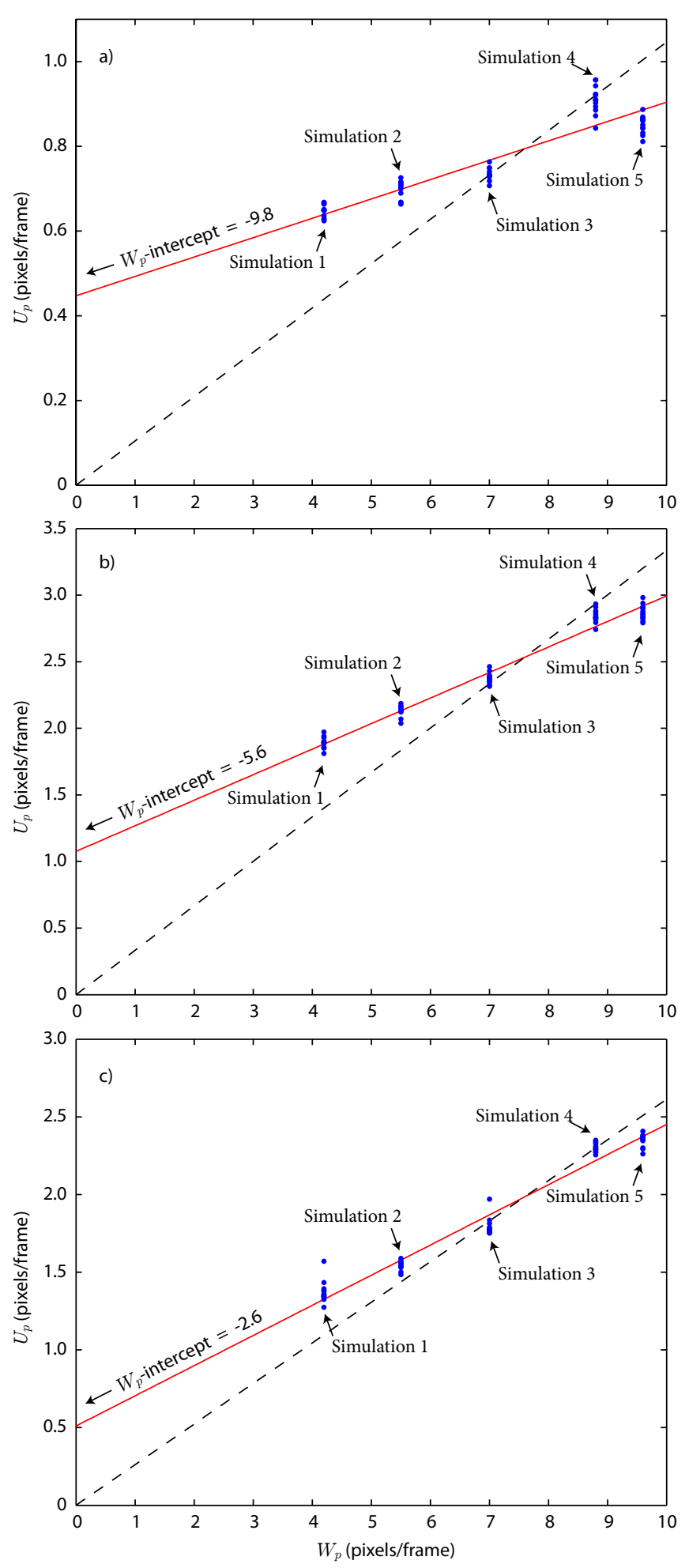

Fig. 10 Flow rate metrics from the five simulations compared to measured flow for a) region-based matching, b) Hovmöller diagram analysis, and c) temporal cross-correlation. Zero-crossing and non-zero-crossing regressions are shown. The $W_{p^{-}}$ intercept value is also indicated. 


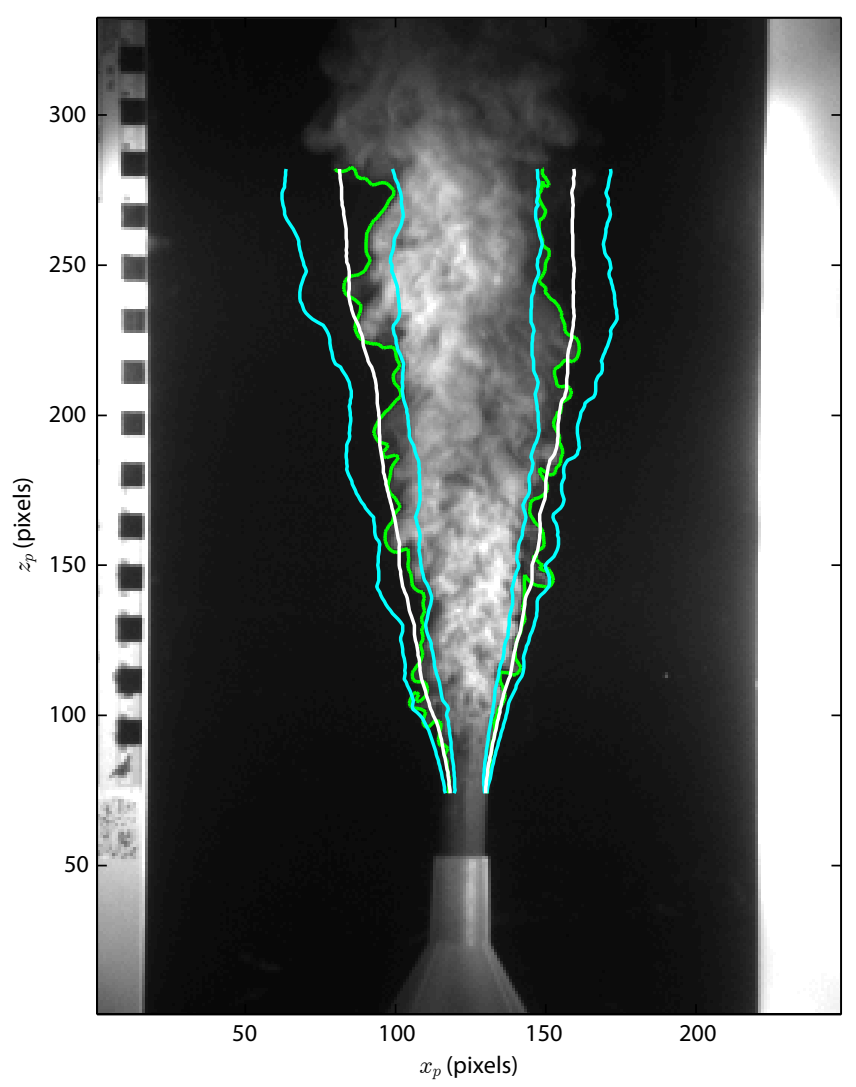

Fig. 11 Single plume edge position (green lines), along with the time-averaged mean plume edge position (white lines), and two standard deviations away from the time-averaged means (blue lines). The standard deviation of the plume edges increases with increasing distance from the nozzle. We normalize the standard deviations by the plume half-width and average all the values together to obtain a single measure of the plume edge position variance for each 30 -s video sequence. 


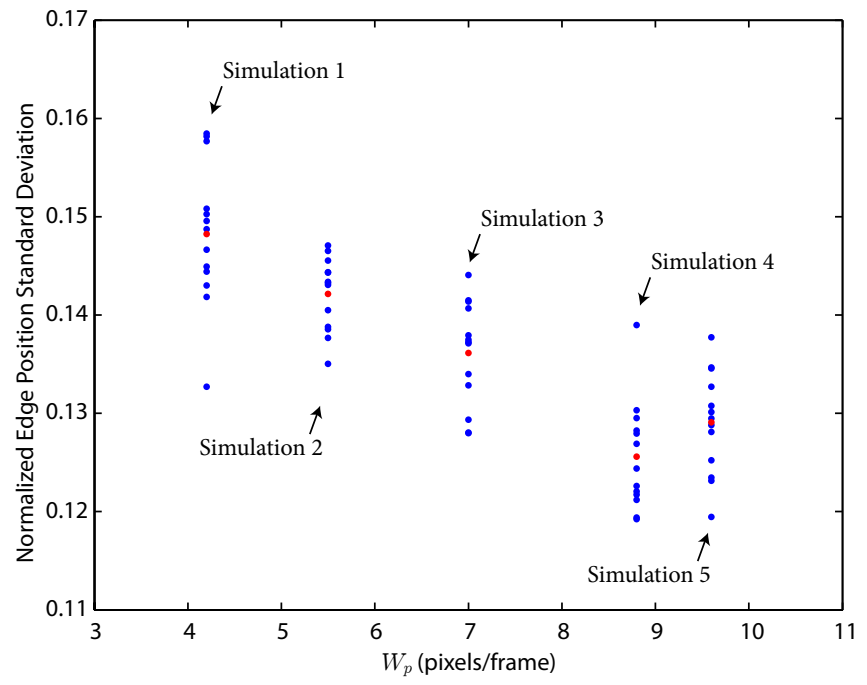

Fig. 12 Average plume edge standard deviations, normalized to the plume half-width, as a function of nozzle flow rate, for 13 30-s video sequences collected during five simulations. The red dots show the average standard deviation for each simulation. Increasing flow rate tends to increase the variance of the plumes edge, suggesting that deeper parts of the plume are imaged at slower nozzle flow rates.

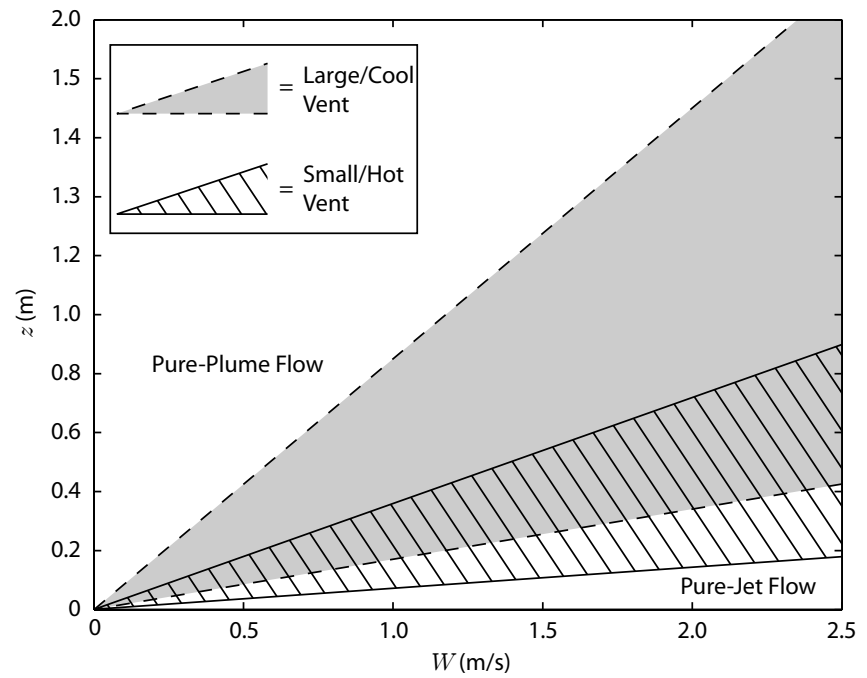

Fig. 13 Transition regions for two black smokers as a function of average nozzle velocity, $W$, and distance from the nozzle, $z$. For a given nozzle velocity, the transition region increases with size and begins farther from the nozzle with increasing nozzle size and decreasing $\Delta \rho$. Here we show the transition regions for a large (Diameter $=0.08 \mathrm{~m}), \operatorname{cool}\left(\Delta \rho=250 \mathrm{~kg} / \mathrm{m}^{3}\right)$ black smoker, and a small (Diameter $=0.02 \mathrm{~m})$, hot $\left(\Delta \rho=350 \mathrm{~kg} / \mathrm{m}^{3}\right)$ black smoker. 